\title{
Slip Effects on the Flow of a Second Grade Fluid in a Varying Width Channel with Application to Stenosed Artery
}

\author{
Abdul Majeed Siddiqui ${ }^{1}$, Tahira Haroon ${ }^{2}$, Zarqa Bano ${ }^{2, *}$ \\ ${ }^{1}$ Department of Mathematics, York Campus, Pennsylvania State University, York, USA \\ ${ }^{2}$ Department of Mathematics, COMSATS Institute of Information Technology, Park Road, Chak Shehzad Islamabad, 44000,Pakistan \\ *Corresponding Author: xarqa@hotmail.com
}

Copyright (C)2013 Horizon Research Publishing All rights reserved.

\begin{abstract}
This article examines the slip effect on the creeping flow of an incompressible second grade fluid in an axisymmetric channel having varying width. We have used three different methods depending upon the geometrical configuration to find out the solution. The results obtained are applied to study the flow of a second grade fluid through a smooth stenosis. To understand the flow behavior near stenosis, resistance to the flow and shear stress at the wall are calculated. The results obtained are graphically evaluated for different values of dimensionless non-Newtonian parameters, maximum height of the stenosis and slip parameter. It is observed that the resistance to the flow and wall shear stress increase with increasing value of non-Newtonian parameter and maximum height of the stenosis and decrease as the value of slip parameter increases.
\end{abstract}

Keywords Second grade fluid, Slip, Varying Width, Resistance to flow.

\section{Introduction}

Stenosis is formed due to deposition of fatty substance on the inner sides of arteries. This abnormal growth may get worse which can cause severe disorder in the blood flow system and may lead to various arterial diseases. The formation, progression and development of arterial stenosis depend upon hydrodynamic factors of the blood. The rheological and fluid dynamic properties of blood and its flow could play a vital role in fundamental understanding, diagnosis and treatment of many cardiovascular diseases [1]. The rheological properties of blood being very important from the point of view of basic understanding of arterial stenosis many researchers have studied blood flow through stenosed arteries both theoretically and experimentally. Out of these studies the work done experimentally by [2] and theoretically by [3] are commendable.

It is observed that the flow patterns in many applications show a slip of fluid particles, that is the fluid fails to adhere to the wall resulting in the slide of fluid particles along the wall. Many authors have studies the fluid behavior of suspensions in general and blood flow in particular both theoretically [4-7] and experimentally [8, 9] and have observed a slip of blood particles at the flow boundaries. Thus the consideration of slip at the constricted wall seems to be quite realistic in the modeling of blood flow.

A significant amount of interest has been paid to the problem of flow through channels of varying gap and non-uniform pipes with slip effects. Flows through elastic tubes, blood circulation through capillaries are some of them. The flow of a fluid in an elastic tube, without considering slip effects, was first approximated by Rashkevsky [10] and later by Morgan [11]. This problem was further investigated by Langlois [12] under several approximations on the geometry of the gap for a Newtonian fluid. Recently Siddique et al [13] have extended the work of Langlois for a non-Newtonian fluid of second grade with application to stenosed artery. However they did not take into account slip effects in their work.

In this study an attempt has been made to study the effects of slip at the stenotic wall for the creeping flow of an incompressible second grade fluid in an axisymmetric channel having varying width. we have used three different methods depending upon the geometrical configuration to find out the solution. The results obtained are applied to study the flow of a second grade fluid through a smooth constriction. To understand the flow behavior near stenosis, resistance to the flow, shear stress at the wall and stress at the stenosis throat are calculated. The results obtained are numerically evaluated for different values of dimensionless non-Newtonian parameters $\lambda_{1}$ and $\lambda_{2}$ and maximum height of the stenosis $\delta_{m}$ and slip parameter $\gamma$. In the following sections, the problem is formulated, solved and the obtained results are discussed.

\section{Governing Equations}

The primary equations that govern the flow of incompressible fluid in the absence of body forces and thermal 
effects are

$$
\begin{gathered}
\operatorname{div} \mathbf{V}=0, \\
\rho \dot{\mathbf{V}}=\operatorname{div} \mathbf{T}+\rho \mathbf{f},
\end{gathered}
$$

where $\rho$ is the constant density, $\mathbf{V}$ is the velocity vector, dot over $\mathbf{V}$ denotes the material time derivative and $\mathbf{T}$ is the Cauchy stress tensor, which for second grade fluid can be defined as:

$$
\mathbf{T}=-p \mathbf{I}+\mu \mathbf{A}_{\mathbf{1}}+\alpha_{1} \mathbf{A}_{\mathbf{2}}+\alpha_{2} \mathbf{A}_{\mathbf{1}}^{\mathbf{2}},
$$

where in equation (3) $\mu$ is the coefficient of viscosity, $p$ is the pressure, $\alpha_{1}$ and $\alpha_{2}$ are the normal stress modulii and $\mathbf{A}_{\mathbf{1}}$ and $\mathbf{A}_{\mathbf{2}}$ are the first and second Rivlin-Ericksen tensors respectively, defined as:

$$
\begin{array}{r}
\mathbf{A}_{\mathbf{1}}=\mathbf{L}+\mathbf{L}^{\mathbf{T}} ; \quad \mathbf{L}=\nabla \mathbf{V}, \\
\mathbf{A}_{\mathbf{2}}=\dot{\mathbf{A}}_{\mathbf{1}}+\mathbf{A}_{\mathbf{1}} \mathbf{L}+\mathbf{L}^{\mathbf{T}} \mathbf{A}_{\mathbf{1}},
\end{array}
$$

and

$$
(\dot{*})=\frac{\partial(*)}{\partial t}+(\mathbf{V} \cdot \nabla)(*) .
$$

With the help of equations (3) and (4), equation (2) becomes

$$
\begin{aligned}
\rho \dot{\mathbf{V}} & =-\nabla p+\mu \nabla^{2} \mathbf{V}+\left(\alpha_{1}+\alpha_{2}\right) \operatorname{div} \mathbf{A}_{\mathbf{1}}^{\mathbf{2}}+\rho \mathbf{f} \\
& +\alpha_{1}\left[\nabla^{2} \mathbf{V}_{t}+\nabla^{2}(\nabla \times \mathbf{V}) \times \mathbf{V}+\nabla\left(\mathbf{V} \cdot \nabla^{2} \mathbf{V}\right.\right. \\
& \left.\left.+\frac{1}{4}\left|\mathbf{A}_{\mathbf{1}}\right|^{2}\right)\right] .
\end{aligned}
$$

\section{Problem Statement}

We consider creeping flow of an incompressible second grade fluid through an infinitely long horizontal channel of smoothly varying gap when wall slippage between the channel wall is possible. We use Cartesian coordinate system, taking $x$-axis in the direction of the flow and $y$ axis perpendicular to it. For simplicity we assume that the channel is symmetric with respect to $x$-axis. In the case of plane steady flow, we take

$$
\mathbf{V}=[u(x, y), v(x, y)],
$$

equation (1) gives

$$
\frac{\partial u}{\partial x}+\frac{\partial v}{\partial y}=0
$$

and momentum balance equation (6) in component form yields

$$
\begin{gathered}
-\rho v \Omega=-\frac{\partial \hat{p}}{\partial x}-\mu \frac{\partial \Omega}{\partial y}-\alpha_{1} v \nabla^{2} \Omega, \\
\rho u \Omega=-\frac{\partial \hat{p}}{\partial y}+\mu \frac{\partial \Omega}{\partial x}+\alpha_{1} u \nabla^{2} \Omega,
\end{gathered}
$$

where $\Omega$ is the vorticity defined as

$$
\Omega=\frac{\partial v}{\partial x}-\frac{\partial u}{\partial y}
$$

and

$$
\hat{p}(x, y)=p+\frac{\rho}{2}\left(u^{2}+v^{2}\right)-\alpha_{1}\left\{u \nabla^{2} u+v \nabla^{2} v\right\}
$$

$$
-\frac{1}{4}\left(3 \alpha_{1}+2 \alpha_{2}\right)\left|A_{1}^{2}\right|
$$

The walls of the channel are bounded below and above by the curve

$$
y= \pm h(x),
$$

where $h(x)$ is smooth and positive for all values of $x$. Since for creeping flow the convective part of momentum equation is negligibly small, equations (9) and (10) reduce to

$$
\frac{\partial \hat{p}}{\partial x}=-\mu \frac{\partial \Omega}{\partial y}-\alpha_{1} v \nabla^{2} \Omega
$$

$$
\frac{\partial \hat{p}}{\partial y}=-\mu \frac{\partial \Omega}{\partial x}+\alpha_{1} u \nabla^{2} \Omega
$$

where

$\hat{p}(x, y)=p-\alpha_{1}\left\{u \nabla^{2} u+v \nabla^{2} v\right\}-\frac{1}{4}\left(3 \alpha_{1}+2 \alpha_{2}\right)\left|A_{1}^{2}\right|$,

and

$$
\left|A_{1}^{2}\right|=8\left|\frac{\partial u}{\partial x}\right|^{2}+2\left(\frac{\partial u}{\partial y}+\frac{\partial v}{\partial x}\right)^{2}
$$

The associated slip boundary conditions are

$u[x, \pm h(x)] \mp \gamma T_{x y}=v[x, \pm h(x)] \mp \gamma T_{x y}=0, \quad$ for all $x$.

Eliminating the pressure between equations (14) and (15) we have a third order differential equation for $u$ and $v$. Hence in addition to the no-slip boundary condition (18) we need one more boundary condition. This boundary condition is obtained by calculating the net flux $Q$ across the channel, which has to be constant at all cross-sections of the channel for an incompressible second grade fluid. Hence

$$
\int_{-h(x)}^{h(x)} u d y=Q, \quad \text { a constant for all } x
$$

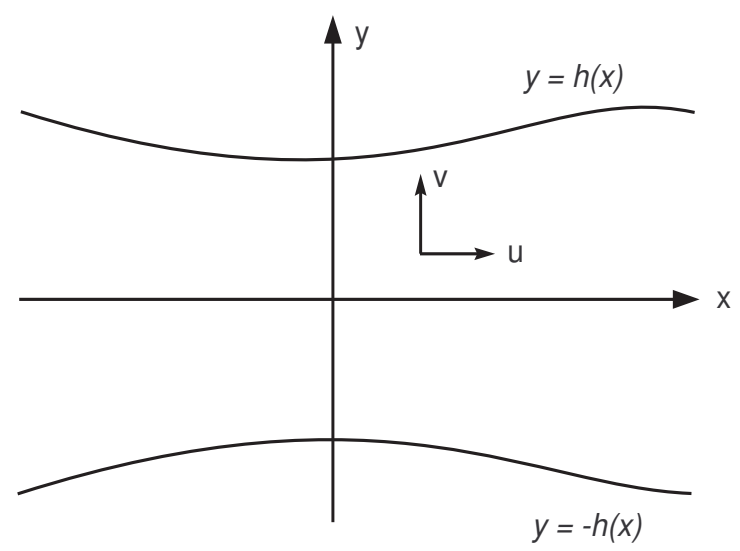

Figure 1. Flow in varying width channel 


\section{Solution of the Problem}

The solution of the problem is found using the three approximate methods depending upon the three different physical situations. Equations (8), (14) and (15) are three partial differential equations for three unknown functions $u, v$ and $p$. Once the velocity field is determined the pressure field (16) can be calculated by integrating equations (14) and (15). When suitable restrictions are placed upon $h(x)$ the boundary value problem represented by equations (8), (14) and (15) together with the boundary conditions (18) and (19) is precisely of the sort in which it is helpful to look at the local picture of flow.

\subsection{Method 1:Negligible Wall Slope:}

If slope $h^{\prime}(x)$ is small everywhere compared with unity, it is reasonable to assume that, at each value of $x$ the component of velocity and pressure gradient are approximately equal to those obtaining in a channel of uniform width, so there is no variation in $u$ component of velocity in $x$-direction and $v=0$. Thus equations (14) and (15) reduce to

$$
\begin{gathered}
\frac{\partial \hat{p}}{\partial x}=\mu \frac{\partial^{2} u}{\partial y^{2}}, \\
\frac{\partial \hat{p}}{\partial y}=0, \quad v=0
\end{gathered}
$$

where

$$
\hat{p}=p-\alpha_{1} u \frac{\partial^{2} u}{\partial y^{2}}-\frac{1}{2}\left(3 \alpha_{1}+2 \alpha_{2}\right)\left(\frac{\partial u}{\partial y}\right)^{2} .
$$

Solving equation (20) subject to equation (18), we find that

$$
u=\frac{1}{2 \mu}\left(-\frac{\partial \hat{p}}{\partial x}\right)\left(h^{2}-y^{2}+2 \gamma \mu h\right) .
$$

Using the value of $u$ from equation (23) in equation (19), we obtain

$$
Q=-\frac{2}{3 \mu}\left(\frac{\partial \hat{p}}{\partial x}\right)\left(h^{3}+3 \gamma h^{2}\right),
$$

and in terms of flow rate $Q, u$ becomes

$$
u=\frac{3 Q}{4} \frac{\left(h^{2}-y^{2}+2 \gamma h\right)}{\left(h^{3}+3 \gamma h^{2}\right)},
$$

and pressure distribution from equations (16) with the help of equations (14) and (15) yields

$$
\begin{aligned}
p= & -\frac{3}{2} \mu Q \int_{x}^{C} \frac{d x}{\left(h^{3}+3 \gamma h^{2}\right)}-\frac{9 \alpha_{1} Q^{2}\left(h^{2}-y^{2}+2 \gamma h\right)}{8\left(h^{3}+3 \gamma h^{2}\right)^{2}} \\
& +\frac{9 Q^{2}\left(3 \alpha_{1}+2 \alpha_{2}\right) y^{2}}{8\left(h^{3}+3 \gamma h^{2}\right)^{2}} .
\end{aligned}
$$

Direct substitution of velocity profile (25) into (18) reveals that the boundary conditions are exactly satisfied. The equations (8),(14) and (15) are satisfied by velocity profile (25) and pressure distribution (26) provided we neglect terms involving $h^{\prime}(x)$ and $h(x) h^{\prime \prime}(x)$. The validity of this approach therefore requires that

$$
\begin{aligned}
& \left|h^{\prime}(x)\right|<<1, \\
& \left|h(x) h^{\prime \prime}(x)\right|<<1 .
\end{aligned}
$$

From equations (25) and (26) we recover

- solution obtained by Langlois as $\gamma \rightarrow 0$ and by setting $\alpha_{1}=\alpha_{2}=0$.

- solution obtained by Siddiqui et al as $\gamma \rightarrow 0$.

\subsection{Method 2: Negligible Wall Curvature:}

We can remove the restriction on wall slope by assuming that the channel width $h(x)$ is a linear function of $x$. Depending upon the sign of $h^{\prime}(x)$ we have different geometrical situations of the channel. If $h^{\prime}(x)$ is positive, the channel is approximated by a divergent wedge with a source of flux $\mathrm{Q}$ at its vortex. If $h^{\prime}(x)$ is negative, the wedge is convergent with a sink at its vortex. This analysis is carried out assuming $h^{\prime}(x)$ as positive and similar results can be obtained when $h^{\prime}(x)$ is negative. The equations of motion for the fully developed creeping flow of a second grade fluid in plane polar coordinates are

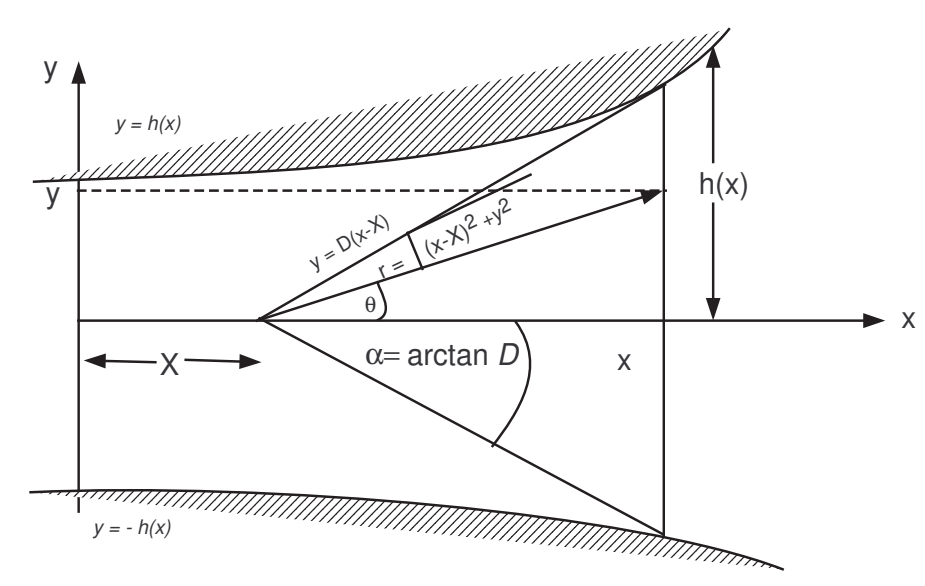

Figure 2. Wedge flow geometry

$$
\frac{\partial}{\partial r}(r u)+\frac{\partial v}{\partial \theta}=0
$$

$$
\begin{aligned}
& \frac{\partial \hat{p}}{\partial r}=\mu\left[\nabla^{2} u-\frac{u}{r^{2}}-\frac{2}{r^{2}} \frac{\partial v}{\partial \theta}\right]-\alpha_{1} v \nabla^{2} \Omega, \\
& \frac{\partial \hat{p}}{\partial \theta}=\mu r\left[\nabla^{2} v-\frac{v}{r^{2}}+\frac{2}{r^{2}} \frac{\partial u}{\partial \theta}\right]+\alpha_{1} r u \nabla^{2} \Omega
\end{aligned}
$$

where $\hat{p}$ is modified pressure and is defined as

$$
\begin{aligned}
\hat{p} & =p-\left[\alpha _ { 1 } \left\{u\left(\nabla^{2} u-\frac{u}{r^{2}}-\frac{2}{r^{2}} \frac{\partial v}{\partial \theta}\right)+v\left(\nabla^{2} v\right.\right.\right. \\
& \left.\left.\left.-\frac{v}{r^{2}}+\frac{2}{r^{2}} \frac{\partial u}{\partial \theta}\right)\right\}+\frac{\left(3 \alpha_{1}+2 \alpha_{2}\right)}{4}\left|\mathbf{A}_{1}^{2}\right|\right],
\end{aligned}
$$

and

$$
\nabla^{2}=\frac{\partial^{2}}{\partial r^{2}}+\frac{1}{r} \frac{\partial}{\partial r}+\frac{1}{r^{2}} \frac{\partial}{\partial \theta}
$$




$$
\Omega=\frac{\partial v}{\partial r}+\frac{v}{r}-\frac{1}{r} \frac{\partial u}{\partial \theta} .
$$

The slip boundary conditions for the wedge flow are

$$
\begin{aligned}
& u(r, \pm \alpha) \mp \gamma T_{r \theta}=v(r, \pm \alpha) \mp \gamma T_{r \theta}=0, \\
& \int_{-\alpha}^{\alpha} r u d \theta=Q .
\end{aligned}
$$

Introducing the following dimensionless variables

$$
\begin{aligned}
\bar{p}=\frac{p}{\rho \omega^{2}}, \quad \bar{u}=\frac{u}{\omega}, \quad \bar{v} & =\frac{v}{\omega}, \quad \bar{r}=\frac{r}{\beta}, \quad \bar{Q}=\frac{Q}{\beta \omega}, \\
\overline{T_{r \theta}} & =\frac{T_{r \theta} \beta}{\mu \omega},
\end{aligned}
$$

where the bars denote dimensionless quantities. Equations (28)-(34) using (46) and after neglecting bars, become

$$
\begin{aligned}
& \frac{\partial}{\partial r}(r u)+\frac{\partial v}{\partial \theta}=0 \\
& \frac{\partial \hat{p}}{\partial r}=\left[\nabla^{2} u-\frac{u}{r^{2}}-\frac{2}{r^{2}} \frac{\partial v}{\partial \theta}\right]-\lambda_{1} v \nabla^{2} \Omega \\
& \frac{\partial \hat{p}}{\partial \theta}=r\left[\nabla^{2} v-\frac{v}{r^{2}}+\frac{2}{r^{2}} \frac{\partial u}{\partial \theta}\right]+\lambda_{1} r u \nabla^{2} \Omega(38) \\
& \hat{p}=\operatorname{Rep}-\left[\lambda _ { 1 } \left\{u\left(\nabla^{2} u-\frac{u}{r^{2}}-\frac{2}{r^{2}} \frac{\partial v}{\partial \theta}\right)+v\left(\nabla^{2} v\right.\right.\right. \\
& \left.\left.\left.-\frac{v}{r^{2}}+\frac{2}{r^{2}} \frac{\partial u}{\partial \theta}\right)\right\}+\frac{\left(3 \lambda_{1}+2 \lambda_{2}\right)}{4}\left|\mathbf{A}_{1}^{2}\right|\right] \\
& \nabla^{2}=\frac{\partial^{2}}{\partial r^{2}}+\frac{1}{r} \frac{\partial}{\partial r}+\frac{1}{r^{2}} \frac{\partial}{\partial \theta} \\
& \Omega=\frac{\partial v}{\partial r}+\frac{v}{r}-\frac{1}{r} \frac{\partial u}{\partial \theta}
\end{aligned}
$$

where $R e=\frac{\omega \beta}{\nu}$ is the Reynolds number, and $\lambda_{1}=\frac{\alpha_{1} \omega}{\mu \beta}$ and $\lambda_{2}=\frac{\alpha_{2} \omega}{\mu \beta}$ are non-Newtonian dimensionless numbers.

The dimensionless slip boundary conditions become:

$$
\begin{aligned}
& u(r, \pm \alpha) \mp \gamma T_{r \theta}=v(r, \pm \alpha) \mp \gamma T_{r \theta}=0, \\
& \int_{-\alpha}^{\alpha} r u d \theta=Q .
\end{aligned}
$$

where $\gamma=\frac{\gamma \mu}{\beta}$ is the dimensionless slip parameter. In order to solve the problem we assume that the radial component of velocity is of the form $u=\frac{f(\theta)}{r}$. Under this assumption the continuity equation (28) gives $\frac{\partial v}{\partial \theta}=0$. To satisfy the slip boundary conditions, $v$ should be zero everywhere in the velocity field. Thus

$$
u=\frac{f(\theta)}{r}, \quad v=0 .
$$

Equations (29)-(32) using profile (43) and eliminating pressure gradient, yield

$$
\frac{1}{r^{3}}\left(f^{\prime \prime \prime}+4 f^{\prime}\right)-\frac{4 \alpha_{1}}{r^{5}}\left(4 f f^{\prime \prime \prime}+4 f f^{\prime}\right)=0 .
$$

From equation (44) we have either

$$
\frac{1}{r^{3}}\left(f^{\prime \prime \prime}+4 f^{\prime}\right)=0,
$$

or

$$
\frac{4 \lambda_{1}}{r^{5}}\left(4 f f^{\prime \prime \prime}+4 f f^{\prime}\right)=0 .
$$

Solving equation (45) using the boundary conditions from equations (34), we obtain

$$
f=\frac{Q\left(\sin ^{2} \alpha-\sin ^{2} \theta+2 \gamma \sin \alpha \cos \alpha\right)}{\left(\sin \alpha \cos \alpha-\alpha+2 \alpha \sin ^{2} \alpha+4 \gamma \sin \alpha \cos \alpha\right)} .
$$

It can be easily verified that equation (47) satisfy equation (46). Tanner [14] had shown that any plane creeping Newtonian velocity filed is also a solution for second grade fluid under identical velocity boundary conditions. So we can conclude that equation (47) is also a solution of equation (44). Using equation (47) into equation (43), we obtain

$$
u=\frac{Q}{r} \frac{\left(\sin ^{2} \alpha-\sin ^{2} \theta+2 \gamma \sin \alpha \cos \alpha\right)}{\left(\sin \alpha \cos \alpha-\alpha+2 \alpha \sin ^{2} \alpha+4 \gamma \sin \alpha \cos \alpha\right)},
$$

$$
v=0
$$

$$
\begin{aligned}
R e \frac{\partial p}{\partial r}= & \frac{-2 Q}{r^{3}} \frac{\left(\cos ^{2} \theta-\sin ^{2} \theta\right)}{G}-\frac{8 Q^{2}\left(3 \lambda_{1}+2 \lambda_{2}\right)}{r^{5} G^{2}} \\
\times & {\left[\left(\sin ^{2} \alpha-\sin ^{2} \theta+2 \gamma \sin \alpha \cos \alpha\right)^{2}\right.} \\
+ & \left.\sin ^{2} \theta \cos ^{2} \theta\right]+\frac{8 Q^{2} \lambda_{1}}{r^{5} G^{2}}\left[\left(\sin ^{2} \alpha-\sin ^{2} \theta\right.\right. \\
+ & \left.2 \gamma \sin \alpha \cos \alpha)\left(\cos ^{2} \theta-\sin ^{2} \theta\right)\right] \\
\operatorname{Re} \frac{\partial p}{\partial \theta} & =\frac{-4 Q}{r^{2}} \frac{(\sin \theta \cos \theta)}{G}-\frac{4 Q^{2}\left(3 \lambda_{1}+2 \lambda_{2}\right)}{r^{4} G^{2}} \\
& \times\left[2\left(\sin ^{2} \alpha-\sin ^{2} \theta+2 \gamma \sin \alpha \cos \alpha\right)\right. \\
& \left.-\left(\cos ^{2} \theta-\sin ^{2} \theta\right)\right] \sin \theta \cos \theta+\frac{4 Q^{2} \lambda_{1}}{r^{4} G^{2}} \\
& \times\left[2\left(\sin ^{2} \alpha-\sin ^{2} \theta+2 \gamma \sin \alpha \cos \alpha\right)\right. \\
& \left.+\left(\cos ^{2} \theta-\sin ^{2} \theta\right)\right] \sin \theta \cos \theta
\end{aligned}
$$

and

$$
\begin{aligned}
R e p & =\frac{Q}{r^{2}} \frac{\left(\cos ^{2} \theta-\sin ^{2} \theta\right)}{G}+\frac{2 Q^{2}\left(3 \lambda_{1}+2 \lambda_{2}\right)}{r^{4} G^{2}} \\
& \times\left[\left(\sin ^{2} \alpha-\sin ^{2} \theta+2 \gamma \sin \alpha \cos \alpha\right)^{2}\right. \\
& \left.+\sin ^{2} \theta \cos ^{2} \theta\right]-\frac{2 Q^{2} \lambda_{1}}{r^{4} G^{2}}\left[\left(\sin ^{2} \alpha-\sin ^{2} \theta\right.\right. \\
& \left.+2 \gamma \sin \alpha \cos \alpha)\left(\cos ^{2} \theta-\sin ^{2} \theta\right)\right]+C .
\end{aligned}
$$

where $C$ is constant of integration and $G=(\sin \alpha \cos \alpha-$ $\alpha+2 \alpha \sin ^{2} \alpha+4 \gamma \sin \alpha \cos \alpha$ ) Equations (48)-(51) satisfy the boundary conditions and differential equations (28)(34).

Equations (48)-(52) reduce to

- solution obtained by Langlois as $\gamma \rightarrow 0$ and by setting $\alpha_{1}=\alpha_{2}=0$.

- solution obtained by Siddiqui et al as $\gamma \rightarrow 0$.

In order to use these results in varying width channel problem, we convert them to cartesian coordinates, with the following notation indicated in Figure 2. 


$$
\begin{aligned}
& u=u_{r} \cos \theta, \quad \cos \theta=\frac{(x-X)}{r}, \\
& v=u_{r} \sin \theta, \quad \sin \theta=\frac{y}{r} \\
& \sin \alpha=\frac{D}{\sqrt{1+D^{2}}} \\
& \cos \alpha=\frac{1}{\sqrt{1+D^{2}}}
\end{aligned}
$$

where

$$
\begin{aligned}
& \tan \alpha=D, \quad r=\sqrt{(x-X)^{2}+y^{2}} . \\
& h=D(x-X)
\end{aligned}
$$

The components of pressure gradient are given by

$$
\begin{aligned}
& \frac{\partial p}{\partial x}=\frac{(x-X)}{r} \frac{\partial p}{\partial r}-\frac{y}{r} \frac{\partial p}{\partial \theta} \\
& \frac{\partial p}{\partial y}=\frac{y}{r} \frac{\partial p}{\partial r}+\frac{(x-X)}{r} \frac{\partial p}{\partial \theta} .
\end{aligned}
$$

Equations (48)-(51) using equations (53)-(55) take the following form

$$
\begin{aligned}
& u=\frac{Q D^{3} h}{E} \frac{h^{2}-y^{2}+2 \frac{\gamma}{D}\left(h^{2}+D^{2} y^{2}\right)}{\left(h^{2}+D^{2} y^{2}\right)^{2}}, \\
& v=\frac{Q D^{4} y}{E} \frac{h^{2}-y^{2}+2 \frac{\gamma}{D}\left(h^{2}+D^{2} y^{2}\right)}{\left(h^{2}+D^{2} y^{2}\right)^{2}} \text {, } \\
& \frac{\partial p}{\partial x}=\frac{-2 Q h\left(1+D^{2}\right)}{E} \frac{h^{2}-3 D^{2} y^{2}}{\operatorname{Re}\left(h^{2}+D^{2} y^{2}\right)^{3}} \\
& -\frac{4 Q^{2}\left(3 \lambda_{1}+2 \lambda_{2}\right) h D^{7}}{E^{2} \operatorname{Re}\left(h^{2}+D^{2} y^{2}\right)^{4}}\left[2 D^{2} h^{2}-D^{4} y^{2}+3 y^{2}\right. \\
& +\quad 4 \gamma D\left\{2 h^{2}-3 y^{2}-D^{2} y^{2}+2 \gamma D\left(h^{2}\right.\right. \\
& \left.\left.\left.+\quad D^{2} y^{2}\right)\right\}\right]+\frac{4 Q^{2} \lambda_{1} h\left(1+D^{2}\right) D^{6}}{E^{2} \operatorname{Re}\left(h^{2}+D^{2} y^{2}\right)^{5}}\left[2 D h^{4}\right. \\
& +\quad D^{5} y^{4}-3 D y^{2} h^{2}-5 D^{3} y^{2} h^{2}+5 D^{3} y^{2} \\
& \left.+\quad 4 \gamma D\left(h^{2}+D^{2} y^{2}\right)\left(h^{2}-2 D^{2} y^{2}\right)\right] \text {, } \\
& \frac{\partial p}{\partial y}=\frac{-2 Q y\left(1+D^{2}\right) D^{4}}{E} \frac{3 h^{2}-D^{2} y^{2}}{\operatorname{Re}\left(h^{2}+D^{2} y^{2}\right)^{3}} \\
& -\frac{4 Q^{2}\left(3 \lambda_{1}+2 \lambda_{2}\right) y D^{6}}{E^{2} \operatorname{Re}\left(h^{2}+D^{2} y^{2}\right)^{4}}\left[3 D^{4} h^{2}-h^{2}+2 D^{2} y^{2}\right. \\
& +\quad 4 \gamma D\left\{3 D^{2} h^{2}-2 D^{2} y^{2}+h^{2}+2 \gamma D\left(h^{2}\right.\right. \\
& \left.\left.\left.+\quad D^{2} y^{2}\right)\right\}\right]+\frac{4 Q^{2} \lambda_{1} y\left(1+D^{2}\right) D^{6}}{E^{2} \operatorname{Re}\left(h^{2}+D^{2} y^{2}\right)^{5}}\left[5 D^{2} h^{4}\right. \\
& \text { - } 5 D^{2} y^{2} h^{2}-3 D^{4} y^{2} h^{2}+2 D^{4} y^{4}+h^{4} \\
& \left.+\quad 4 \gamma D\left\{\left(h^{2}+D^{2} y^{2}\right)\left(2 h^{2}-D^{2} y^{2}\right)\right\}\right] \text {. }
\end{aligned}
$$

where

$$
\begin{aligned}
E & =\left(\sin \alpha \cos \alpha-\alpha+2 \alpha \sin ^{2} \alpha+2 \alpha \gamma \sin ^{2} \alpha\right)\left(1+D^{2}\right), \\
& =D-\left(1-(1-2 \gamma) D^{2}\right) \tan ^{-1}(D) .
\end{aligned}
$$

Upon checking by direct substitution, we find that the velocity components given by equation (56) satisfy the boundary conditions given in equation (34). We also find that equation (56) and (58) satisfy the differential equations (8), (14) and (15), provided that

$$
\left|h(x) h^{\prime \prime}(x)\right|<<1,
$$

$$
\left|h(x)^{2} h^{\prime \prime \prime}(x)\right|<<1
$$

If equation (60) is satisfied, it can be verified that

$$
d p=\frac{\partial p}{\partial x} d x+\frac{\partial p}{\partial y} d y
$$

is an exact differential when $\frac{\partial p}{\partial x}$ and $\frac{\partial p}{\partial y}$ are given by equations (57) and (58). Therefore

$$
p=\int_{x}^{C} \frac{\partial p}{\partial x} d x+\int_{0}^{y} \frac{\partial p}{\partial y} d y
$$

$$
\begin{aligned}
p & =\frac{-2 Q}{R e} \int_{x}^{C} \frac{D^{3}\left(1+D^{2}\right)}{E h^{3}} d x-\frac{8 Q^{2}\left(3 \lambda_{1}+2 \lambda_{2}\right)}{R e} \\
& \times \int_{x}^{C} \frac{D^{7}}{E^{2} h^{5}} d x\left[D^{2}+4 \gamma D(1+\gamma D)\right]+\frac{8 Q^{2} \lambda_{1}}{R e} \\
& \times \quad \int_{x}^{C} \frac{D^{7}\left(1+D^{2}\right)}{E^{2} h^{5}}(1+4 \gamma) d x-\frac{2 Q\left(1+D^{2}\right) D^{4}}{R e E} \\
& \times \quad \int_{0}^{y} \frac{3 h^{2} y-D^{2} y^{3}}{\left(h^{2}+D^{2} y^{2}\right)^{3}} d y-\frac{4 Q^{2}\left(3 \lambda_{1}+2 \lambda_{2}\right) D^{6}}{R e E^{2}} \\
& \times \quad \int_{0}^{y} \frac{y}{\left(h^{2}+D^{2} y^{2}\right)^{4}}\left[3 D^{4} h^{2}-h^{2}+2 D^{2} y^{2}\right. \\
& +\quad 4 \gamma D^{2}\left\{3 D^{2} h^{2}-2 D^{2} y^{2}+h^{2}+2 \gamma D\left(h^{2}\right.\right. \\
& \left.\left.\left.+\quad D^{2} y^{2}\right)\right\}\right] d y+\frac{4 Q^{2} \lambda_{1}\left(1+D^{2}\right) D^{6}}{R e E^{2}} \\
& \times \quad \int_{0}^{y} \frac{y}{\left(h^{2}+D^{2} y^{2}\right)^{5}}\left[5 D^{2} h^{4}-5 D^{2} h^{2} y^{2}-3 D^{4} h^{2} y^{2}\right. \\
& \left.+\quad 2 D^{4} y^{4}+h^{4}+4 \gamma D\left\{\left(h^{2}+D^{2} y^{2}\right)\left(2 h^{2}-D^{2} y^{2}\right)\right\}\right] d y,
\end{aligned}
$$

where $\mathrm{C}$ is a constant of integration.

Equations (56)-(64) reduce to

- solution obtained by Langlois as $\gamma \rightarrow 0$ and by setting $\alpha_{1}=\alpha_{2}=0$.

- solution obtained by Siddiqui et al as $\gamma \rightarrow 0$.

\subsection{Method 3: Wall Slope Expanded in Power Series:}

Method 2 discussed above gave cumbersome results even for analytically simple form of $h(x)$. It may also happen that the function $h(x)$ satisfy equations (60) for small $h^{\prime}(x)$ but not negligible. So we can modify the condition

$$
\left|D^{n}\right|=\left|h^{\prime}(x)^{n}\right|<<1,
$$

which is satisfied for some positive integer $\mathrm{n}$. For $n>1$ we expand the results of method 2 in power series in $\mathrm{D}$, neglecting the terms of $n=3$ or higher order in $\mathrm{D}$. For this we proceed as follows: Expanding $\tan ^{-1}(D)$ in power series of $\mathrm{D}$, we have

$$
\tan ^{-1}(D)=D-\frac{1}{3} D^{3}+\frac{1}{5} D^{5}+O\left(D^{7}\right) .
$$

The function E given in equation (59) when expanded in powers of $\mathrm{D}$, gives

$$
E=\frac{4}{3} D^{3}\left[1-\frac{2}{5} D^{2}+\frac{3 \gamma}{D}\left(1-\frac{1}{3} D^{2}\right)+O\left(D^{4}\right)\right],
$$


which after some manipulation, becomes

$$
\frac{D^{3}}{E}=\frac{3}{4}\left[1+\frac{2}{5} D^{2}-\frac{3 \gamma}{D}\left(1+\frac{17}{15} D^{2}\right)+O\left(D^{4}\right)\right] .
$$

Similarly the function $E^{2}$ gives

$$
\frac{D^{6}}{E^{2}}=\frac{9}{16}\left[1+\frac{4}{5} D^{2}-\frac{6 \gamma}{D}\left(1+\frac{28}{15} D^{2}\right)+O\left(D^{4}\right)\right] .
$$

The expressions for the velocity components and pressure, expanding in powers of $D$ and neglecting the third or higher order in $\mathrm{D}$, become

$$
\begin{aligned}
& u=\frac{3 Q}{4 h}\left[[ 1 - ( \frac { y } { h } ) ^ { 2 } + \frac { 2 \gamma } { D } ] \left[1-2 D^{2}\left(\frac{y}{h}\right)^{2}+\frac{2}{5} D^{2}\right.\right. \\
& \left.-\frac{3 \gamma}{D}\left(1+\frac{17}{15} D^{2}\right)+6 \gamma D\left(\frac{y}{h}\right)^{2}\right]+2 \gamma(1 \\
& \left.-\quad 3 \gamma)\left(\frac{y}{h}\right)^{2}\right] \text {, } \\
& v=\frac{3 Q D}{4 h}\left[( \frac { y } { h } ) [ 1 - ( \frac { y } { h } ) ^ { 2 } + \frac { 2 \gamma } { D } ] \left[1-\frac{3 \gamma}{D}(1\right.\right. \\
& \left.\left.+\frac{17}{15} D^{2}\right)+6 \gamma D\left(\frac{y}{h}\right)^{2}\right]+2 \gamma(D-3 \gamma(1 \\
& \left.\left.\left.+\frac{17}{15} D^{2}\right)\left(\frac{y}{h}\right)^{2}\right)\right] \text {, } \\
& \frac{\partial p}{\partial x}=\frac{-3 Q}{2 \operatorname{Reh}^{3}}\left[1-6 D^{2}\left(\frac{y}{h}\right)^{2}+\frac{7}{5} D^{2}-3 \gamma\left\{\frac{1}{D}\right.\right. \\
& \left.\left.\times \quad\left(1+\frac{17}{15} D^{2}\right)\left(1+D^{2}\right)-6 D\left(\frac{y}{h}\right)^{2}\right\}\right] \\
& -\frac{9 Q^{2}\left(3 \lambda_{1}+2 \lambda_{2}\right)}{4 \operatorname{Reh}^{5}}\left[\frac{3}{2}\left(\frac{y}{h}\right)^{2}+4 \gamma\left\{1-\frac{3}{2} D\right.\right. \\
& -\frac{15}{4}\left(\frac{y}{h}\right)^{2}-\frac{21}{15} D^{2}\left(\frac{y}{h}\right)^{2}+9 D\left(\frac{y}{h}\right)^{4} \\
& -6 \gamma\left(1+\frac{28}{15} D^{2}-\frac{3}{2}\left(\frac{y}{h}\right)^{2}-\frac{14}{5} D^{2}\left(\frac{y}{h}\right)^{2}\right. \\
& +\quad \gamma D)\}]+\frac{9 Q^{2} \lambda_{1}}{4 \operatorname{Reh}^{5}}\left[\left(1-\frac{3}{2} D\left(\frac{y}{h}\right)^{2}\right) D\right. \\
& -3 \gamma\left\{2\left(1-\frac{1}{3} D+D^{2}\right)-\left(1+\frac{43}{15} D^{2}\right)\right. \\
& \left.\left.\times\left(3\left(\frac{y}{h}\right)^{2}-4 \gamma\right)\right\}\right] \text {, }
\end{aligned}
$$$$
\frac{\partial p}{\partial y}=\frac{-9 Q D}{2 h^{3}}\left(\frac{y}{h}\right)\left(D-3 \gamma\left(1+\frac{17}{15} D^{2}\right)\right)
$$$$
+\frac{9 Q^{2}\left(3 \lambda_{1}+2 \lambda_{2}\right)}{4 \operatorname{Reh}^{5}}\left(\frac{y}{h}\right)\left[1-6 D^{2}\left(\frac{y}{h}\right)^{2}+\frac{4}{5} D^{2}\right.
$$$$
\text { - } 2 \gamma\left\{2 D(1+2 \gamma D)-\frac{3}{D}\left(1-6 D^{2}\left(\frac{y}{h}\right)^{2}\right)\right.
$$$$
\left.\left.-\quad 4 \gamma D(1+2 \gamma D)+\frac{28}{15} D^{2}\right\}\right]+\frac{9 Q^{2} \lambda_{1}}{4 \operatorname{Reh}^{5}}\left(\frac{y}{h}\right)
$$$$
\times \quad\left[1-10 D^{2}\left(\frac{y}{h}\right)^{2}-\frac{29}{5} D^{2}+2 \gamma\{4 D(1\right.
$$$$
\left.+\frac{1}{2} D^{2}\left(\frac{y}{h}\right)^{2}\right)-\frac{3}{D}\left(1+\frac{103}{15} D^{2}-10 D^{2}\left(\frac{y}{h}\right)^{2}\right.
$$$$
\left.\left.\left.+\quad 4 \gamma D\left(1+\frac{1}{2} D^{2}\left(\frac{y}{h}\right)^{2}\right)\right)\right\}\right]
$$

and the equation for the pressure distribution becomes

$$
\begin{aligned}
p & =\frac{3 Q}{4 R e}\left[-2 \int_{x}^{C} \frac{1}{h^{3}}\left(1+\frac{7}{5} D^{2}-3 \gamma\left(\frac{1}{D}(1\right.\right.\right. \\
& \left.\left.\left.+\frac{17}{15} D^{2}\right)-D\right)\right) d x-\frac{3}{h^{2}}\left(\frac{y}{h}\right)^{2}\{D-3 \gamma(1 \\
& \left.\left.\left.+\frac{17}{15} D^{2}\right)\right\}\right]-\frac{9 Q^{2}\left(3 \lambda_{1}+2 \lambda_{2}\right)}{4 R e}\left[2 \int _ { x } ^ { C } \frac { 1 } { h ^ { 5 } } \left[2 \gamma D^{2}\right.\right. \\
& \left.+24 \gamma^{2} D(1+\gamma D)\right] d x+\frac{1}{h^{4}}\left(\frac{y}{h}\right)^{2}\left\{\frac{1}{2}+\frac{2}{5} D^{2}\right. \\
& -\frac{3}{2} D^{2}\left(\frac{y}{h}\right)^{2}-2 \gamma D(1+2 \gamma D)-\frac{6 \gamma}{D}\left\{\frac{1}{2}+\frac{2}{5} D^{2}\right. \\
& \left.\left.\left.+\frac{3}{2} D^{2}\left(\frac{y}{h}\right)^{2}-2 \gamma D(1+2 \gamma D)+\frac{14}{15} D^{2}\right\}\right\}\right] \\
& +\frac{1}{h^{4}}\left(\frac{y}{h}\right)^{2}\left\{\frac{1}{2}+\frac{29}{10} D^{2}-\frac{5}{2} D^{2}\left(\frac{y}{h}\right)^{2} \int_{x}^{C} \frac{1}{h^{5}}\left(D-6 \gamma\left(1+\frac{28}{15} D^{2}\right)\right)(1+4 \gamma)\right. \\
& +4 \gamma D\left(1+D^{2}\left(\frac{y}{h}\right)^{2}\right)-\frac{6 \gamma}{D}\left\{\frac{1}{2}+\frac{103}{30} D^{2}\right. \\
& \left.\left.\left.-\frac{5}{2} D^{2}\left(\frac{y}{h}\right)^{2}+4 \gamma D\left(1+D^{2}\left(\frac{y}{h}\right)^{2}\right)\right\}\right\}\right]
\end{aligned}
$$

Equations (70)-(74) reduce to

- solution obtained by Langlois as $\gamma \rightarrow 0$ and by setting $\alpha_{1}=\alpha_{2}=0$.

- solution obtained by Siddiqui et al as $\gamma \rightarrow 0$.

\subsection{Comparison of the Three Methods:}

The average pressure gradient across the channel is

$$
\triangle p=\frac{-1}{h(x)} \int_{0}^{h(x)} \frac{\partial p}{\partial x} d y
$$

$\triangle p$ is calculated in the neighborhood of a given value of $x$ using the three methods mentioned above and the weight functions are compared. $\triangle p$ is found to be

$$
\triangle p=\frac{3 Q}{2 R e h^{3}} F_{i}(D), \quad \text { for } i=1,2,3
$$

where $F_{i}(D)$, for $i=1,2,3$ are given by:

$$
\begin{aligned}
F_{1}(D) & =\frac{h}{h+3 \gamma}+\frac{\left(3 \lambda_{1}+2 \lambda_{2}\right) D^{2} h^{5} Q}{2\left(h^{3}+3 D^{2} \gamma\right)^{3}}(D+3 \gamma) \\
& -\frac{\lambda_{1} D^{2} Q}{4 h(h+3 \gamma)^{3}}(4 D-3 h+3 \gamma+12 D \gamma \\
& \left.-6 h \gamma+18 \gamma^{2}\right), \\
F_{2}(D)= & \frac{4 D^{3}\left(1+D^{2}\right)}{3\left(1+D^{2}\right)^{2} E}+\frac{D^{5} Q\left(3 \lambda_{1}+2 \lambda_{2}\right)}{6\left(1+D^{2}\right)^{2} h^{2} E}[D \\
\times & (12 \gamma+D(11+D(48 \gamma+D(15+36 D \gamma \\
+ & \left.\left.\left.\left.80 \gamma^{2}+D^{2}\left(9+48 \gamma^{2}\right)\right)\right)\right)-3\right)+3(1 \\
+ & \left.D^{2}\right)^{2}\left(1-4 D \gamma+12 D^{3} \gamma+D^{4}\left(3+16 \gamma^{2}\right)\right] \\
\times & \tan ^{-1}(D)-\frac{D^{7} \lambda_{1} Q}{144 D^{3}\left(1+D^{2}\right)^{4} h^{4} E}\left[45 D h^{2}\right.
\end{aligned}
$$




$$
\begin{aligned}
+ & 3 D^{9}\left(25+48 h^{2}(1+2 \gamma)\right)+D^{5}(365 \\
+ & \left.9 h^{2}(51+224 \gamma)\right)+D^{7}\left(275+3 h^{2}(161\right. \\
+ & 352 \gamma))+3 D^{3}\left(-25+h^{2}(135+416 \gamma)\right) \\
+ & 3\left(1+D^{2}\right)^{4}\left(-15 h^{2}+D^{2}\left(25+48 h^{2}(1\right.\right. \\
+ & \left.2 \gamma))) \tan ^{-1}(D)\right], \\
F_{3}(D) & =1-\frac{3 D^{2}}{5}+\gamma\left(3\left(D-\frac{1}{D}\right)-\frac{17}{5}\left(D^{2}\right.\right. \\
+ & 1))+\frac{3\left(3 \lambda_{1}+2 \lambda_{2}\right) Q}{2 h^{2}}\left[1+\frac{2 \gamma}{15}(18 D\right. \\
& \left.\left.-28 D^{2}-180 \gamma+336 D^{2} \gamma-360 D \gamma^{2}-15\right)\right] \\
& -\frac{3 \lambda_{1} Q}{h^{2}}\left[\frac{D}{2}(2-h)-\frac{\gamma}{5}\left(15-10 D-13 D^{2}\right.\right. \\
+ & \left.\left.60 \gamma+172 D^{2} \gamma\right)\right] .
\end{aligned}
$$

The above three weight functions are graphically evaluated for $\gamma=0,0.01$ and are presented in Figures 4 and 5.

\section{Flow Through a Channel with Smooth Constriction}

The above theory is applied to the problem of flow through a channel with smooth, axisymmetric constriction as shown in Figure 3, defined in non-dimensional variables by [12]

$$
h(x)=H_{0}-\frac{\delta_{m}}{2}\left(1+\cos \frac{\pi x}{x_{0}}\right)
$$

where $\delta_{m}$ is the maximum projection of the constriction and $H_{0}$ is the half width of the channel.

The third approximation method is valid, when the condition given in (65) is satisfied, i-e;

$$
\left|D^{n}\right|=\left|h^{\prime}(x)^{n}\right|<<1,
$$

for some positive integer $n$. The expression for $\mathrm{D}$, using

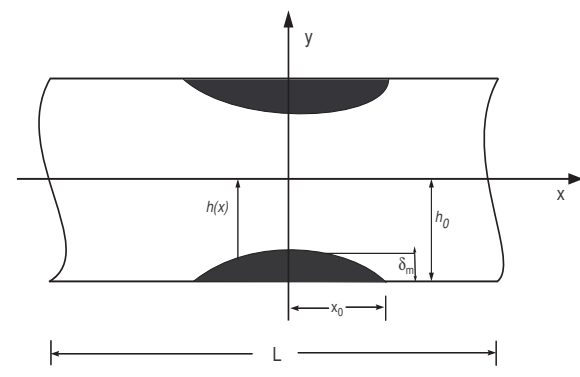

Figure 3. Idealized geometry for stenosis.

equation(80), becomes

$$
D=h^{\prime}(x)=\frac{\pi \delta_{m}}{2 x_{0}} \sin \frac{\pi x}{x_{0}} .
$$

We note that the condition $\left|D^{n}\right|<<1$ will be satisfied if the following non-dimensional quantities take the values

$$
L=4.0,
$$

$$
\begin{aligned}
& x_{0}=1.0, \\
& \delta_{m}=0.32 H_{0}, \\
& H_{0}=1.0 .
\end{aligned}
$$

For these values $|D|$ has a maximum value of 0.5 at $x=x_{0} / 2$ and satisfies the condition given in (65) for all positive values of $n$.

To determine the flow characteristics in the channel near stenosis, it is important to determine the resistance to the flow (RF) and the shear stress at the wall and shear stress at the stenosis throat $\delta_{m}$. The resistance to flow denoted by R.F is defined as:

$$
\text { R.F. }=\frac{\text { average pressure drop across the channel }}{\text { flux in the direction of flow }} .
$$

To determine R.F. we first need to determine average pressure drop across the channel and flux in the direction of flow. The average pressure drop across the channel can be calculated form the following expression

$$
P_{0}-P=\frac{1}{2 h L} \int_{-h(x)}^{h(x)} \int_{0}^{L} \frac{\partial p}{\partial x} d x d y
$$

Using equation (72) and integrating over the interval $-h$ to $+h$ equation(83) take the form

$$
\begin{aligned}
p & =\frac{3 Q}{2 \operatorname{Reh} L} \int_{0}^{L} \frac{1}{h^{2}}\left[1-\frac{3 D^{2}}{5}-\gamma\left(\frac{17}{5}\left(1+D^{2}\right)\right.\right. \\
& \left.\left.+3\left(\frac{1}{D}-D\right)\right)\right] d x+\frac{3 Q^{2}\left(\lambda_{1}+\lambda_{2}\right)}{2 \operatorname{Reh} L} \\
& \times \int_{0}^{L} \frac{1}{h^{4}}\left(\left(\frac{3}{2}-\left(3-\frac{18 D}{5}+\frac{56 D^{2}}{5}\right) \gamma\right.\right. \\
& \left.\left.-\left(36-\frac{336 D^{2}}{5}\right) \gamma^{2}-72 D \gamma^{3}\right)\right) d x+\frac{3 Q^{2} \lambda_{1}}{2 \operatorname{Reh} L} \\
& \times \int_{0}^{L}\left(\frac{3 D^{2}}{2 h^{3}}-\frac{1}{h^{4}}\left(3 D-\left(9-6 D-\frac{39 D^{2}}{5}\right) \gamma\right.\right. \\
& \left.\left.-\left(36+\frac{516 D^{2}}{5}\right) \gamma^{2}\right)\right) d x
\end{aligned}
$$

The dimensionless momentum flux in the horizontal direction has the form

$$
M=\int_{-h}^{h} u^{2} d y
$$

using the expression for $u$ from equation (70) in equation (86) and after performing the indicated integration, we find that

$$
\begin{aligned}
M & =\frac{Q^{2}}{875 h}\left(525+120 D^{2}+64 D^{4}\right)-\frac{3 Q^{2} \gamma}{1750 D^{4} h}\left[112 D^{7}\right. \\
& -23625 \gamma^{3}-2 D^{6}(1143 \gamma-80)-525 D^{2} \gamma(2 \gamma(36 \gamma \\
& -5)-9)-350 D^{3}(\gamma(2+51 \gamma)-1)-10 D^{5}(\gamma \\
& \times(1278 \gamma-169)-202)-5 D^{4}(70+\gamma(28 \gamma(123 \gamma \\
& -20))-857)] .
\end{aligned}
$$

The expression for resistance to the flow become

$$
R . F=\frac{p}{M}
$$

where $p$ and $M$ are given by equations (85) and equation (87) respectively. Analytical evaluation of integral given 
in numerator of equation (85) is complicated therefore we have numerically evaluated it on a computer. The R.F was calculated for different values of slip parameter $\gamma$, non-Newtonian parameters $\lambda_{1}$ and $\lambda_{2}$, and the results are shown in Tables 1-3.

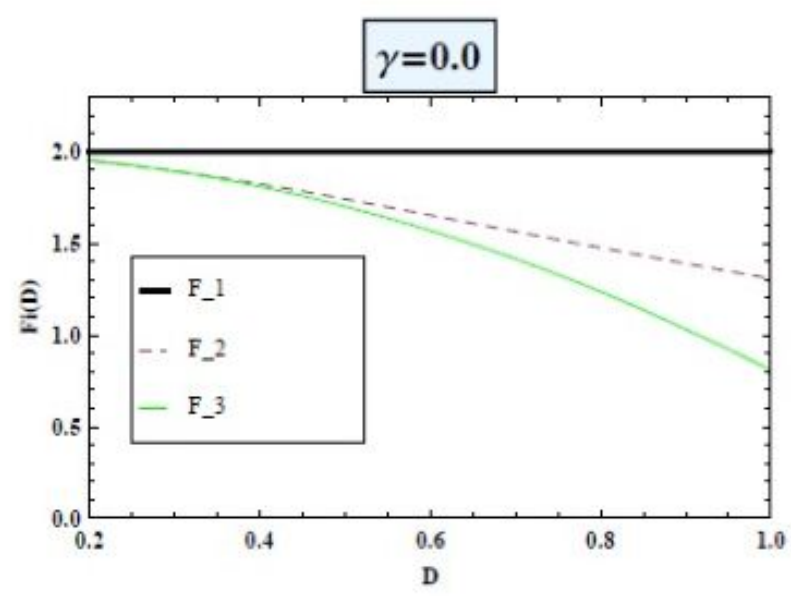

Figure 4. Comparison of Weight Functions for $\gamma=0.0$, while $\lambda_{1}=0.1, \lambda_{2}=0.2$ and $Q=0.02$

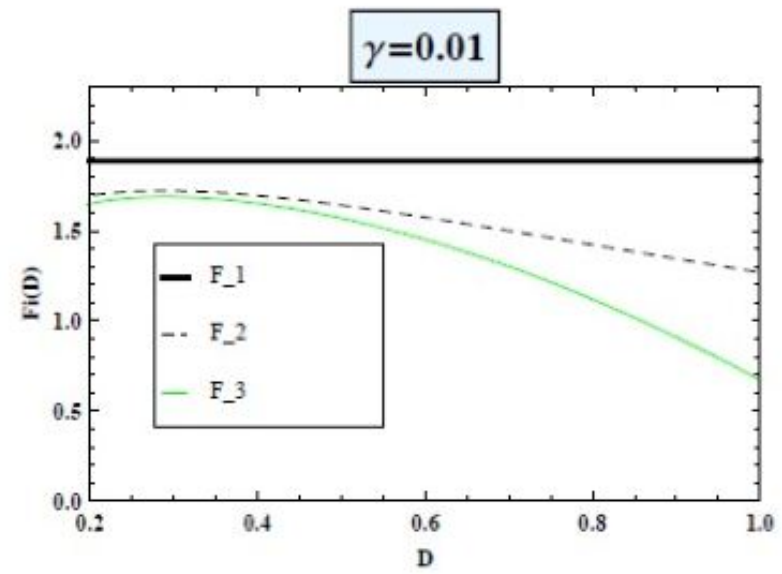

Figure 5. Comparison of Weight Functions for $\gamma=0.0$, while $\lambda_{1}=0.1, \lambda_{2}=0.2$ and $Q=0.02$

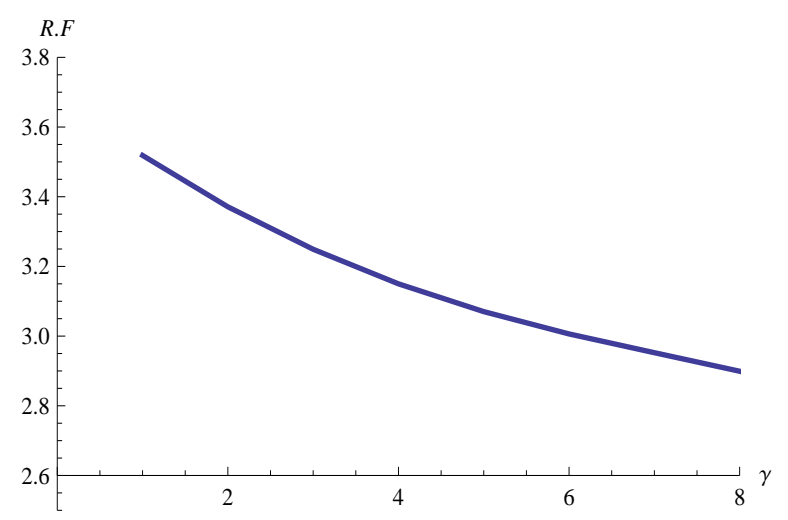

Figure 6. Behaviour of resistance to the flow versus slip parameter
Table 1. Resistance to the flow for different values of $\gamma$, while $\lambda_{1}=0.2, \lambda_{2}=0.02$ and $Q=0.2$

\begin{tabular}{|c|c|c|c|}
\hline$\gamma$ & $\mathrm{h}$ & $\mathrm{D}$ & $\mathrm{R} . \mathrm{F}$ \\
\hline 0. & 0.84 & 0.5 & 3.51856 \\
0.01 & 0.84 & 0.5 & 3.37103 \\
0.02 & 0.84 & 0.5 & 3.2492 \\
0.03 & 0.84 & 0.5 & 3.14999 \\
0.04 & 0.84 & 0.5 & 3.07024 \\
0.05 & 0.84 & 0.5 & 3.0062 \\
0.06 & 0.84 & 0.5 & 2.95238 \\
0.07 & 0.84 & 0.5 & 2.89915 \\
0.08 & 0.84 & 0.5 & 2.82719 \\
0.09 & 0.84 & 0.5 & 2.69327 \\
0.1 & 0.84 & 0.5 & 2.38931 \\
\hline
\end{tabular}

Table 2. Resistance to the flow for different values of $\lambda_{1}$, while $\gamma=0.1, \lambda_{2}=0.2$ and $Q=0.2$

\begin{tabular}{|c|c|c|c|}
\hline$\lambda_{1}$ & $\mathrm{~h}$ & $\mathrm{D}$ & R.F \\
\hline 0. & 0.84 & 0.5 & 1.18504 \\
0.01 & 0.84 & 0.5 & 1.3116 \\
0.02 & 0.84 & 0.5 & 1.43817 \\
0.03 & 0.84 & 0.5 & 1.56473 \\
0.04 & 0.84 & 0.5 & 1.6913 \\
0.05 & 0.84 & 0.5 & 1.81786 \\
0.06 & 0.84 & 0.5 & 1.94442 \\
0.07 & 0.84 & 0.5 & 2.07099 \\
0.08 & 0.84 & 0.5 & 2.19755 \\
0.09 & 0.84 & 0.5 & 2.32411 \\
0.1 & 0.84 & 0.5 & 2.45068 \\
\hline
\end{tabular}

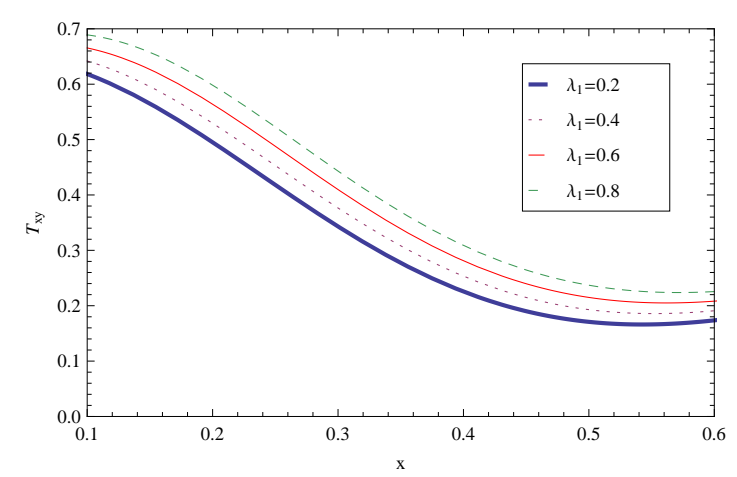

Figure 7. Variation of shear stress for $Q=0.2, \gamma=0.0$ and for different values of $\lambda_{1}$

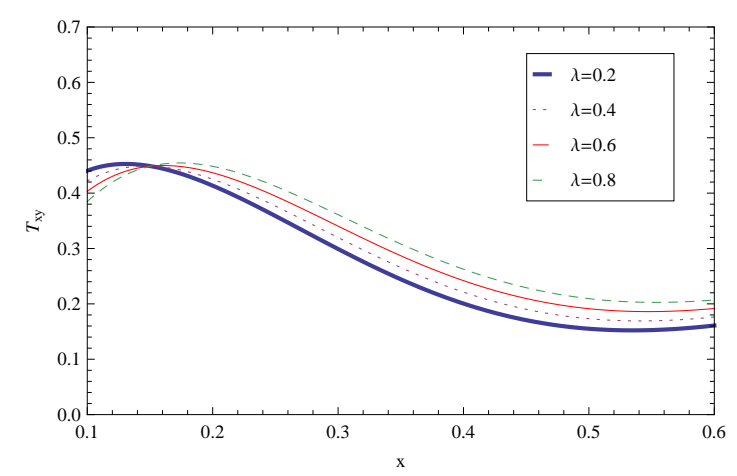

Figure 8. Variation of shear stress for $Q=0.2, \gamma=0.01$ and for different values of $\lambda_{1}$

\section{Results and Discussion}

Three approximate methods based on three different geometrical situations are used to solve two-dimensional 
Table 3. Resistance to the flow for different values of $\lambda_{2}$, while $\gamma=0.1, \lambda_{1}=0.1$ and $Q=0.2$

\begin{tabular}{|c|c|c|c|}
\hline$\lambda_{2}$ & $\mathrm{~h}$ & $\mathrm{D}$ & $\mathrm{R} . \mathrm{F}$ \\
\hline 0. & 0.84 & 0.5 & 0.614483 \\
0.01 & 0.84 & 0.5 & 0.706292 \\
0.02 & 0.84 & 0.5 & 0.798102 \\
0.03 & 0.84 & 0.5 & 0.889912 \\
0.04 & 0.84 & 0.5 & 0.981721 \\
0.05 & 0.84 & 0.5 & 1.07353 \\
0.06 & 0.84 & 0.5 & 1.16534 \\
0.07 & 0.84 & 0.5 & 1.25715 \\
0.08 & 0.84 & 0.5 & 1.34896 \\
0.09 & 0.84 & 0.5 & 1.44077 \\
0.1 & 0.84 & 0.5 & 1.53258 \\
\hline
\end{tabular}

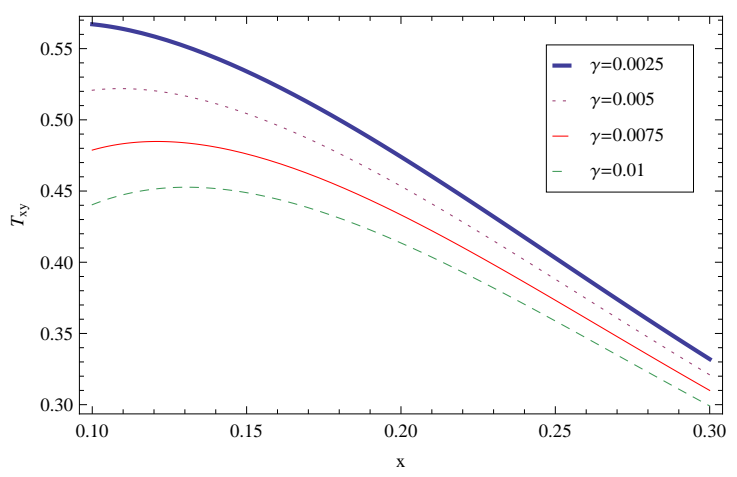

Figure 9. Variation of shear stress for $Q=0.2, \lambda_{1}$ and for different values of $\gamma$

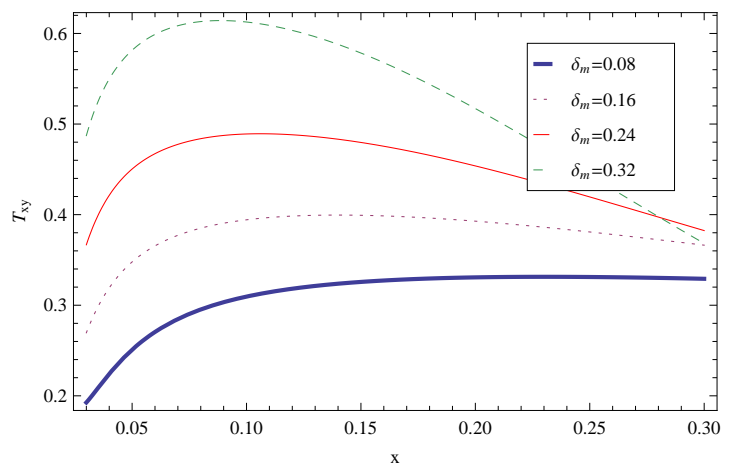

Figure 10. Variation of shear stress for $Q=0.2, \lambda_{1}$ and for different values of $\delta_{m}$

second order nonlinear differential equation arising due to creeping second grade fluid through a channel of varying gap with slip effect. With the help of these methods we are able to have an idea about the geometrical effects of the curved channel on the flow which otherwise are not observed by using other methods. These methods provide an alternate approach to the conventional methods of solving a two dimensional problem. The weight functions $F_{1}, F_{2}$ and $F_{3}$ are calculated for each of the methods and and their behavior is shown in Figure 4 for different values of slip parameter $\gamma$. It is seen that $F_{1}, F_{2}$ and $F_{3}$ are influenced by $\gamma$ and have smaller values than those in the case of no-slip. To study the flow pattern in arteries having stenosis an idealized stenosis geometry is considered and resistance to the flow is determined. The results obtained are numerically evaluated for different values of dimensionless non-Newtonian parameters $\lambda_{1}$, $\lambda_{2}$, and $\gamma$ and are shown in Tables 1 to 3 . It is observed that with increasing the value of $\gamma$ the RF decreases. However opposite behavior is observed with increasing the values of $\lambda_{1}$ and $\lambda_{2}$. Figure 6 shows the behaviour of $\mathrm{RF}$ versus $\gamma$ and it is observed that RF is a decreasing function of $\gamma$. Figures 7 and 8 show the behaviour of shear stress at the wall when $\gamma=0.0$ and $\gamma=0.01$ is fixed and $\lambda_{1}$ is varied respectively. It is observed that the value of shear stress is lesser when $\gamma=0.01$ as compared to that of when $\gamma=0.0$. Figures 9 and 10 show the wall shear stress for different values of $\gamma$ and $\delta_{m}$ repectively. It is observed that shear stress decreases at the wall as we increase the value of $\gamma$; whereas opposite behaviour is observed when the value of $\delta_{m}$ is increased.

\section{Conclusion}

Creeping flow of an incompressible second grade fluid in a channel of varying width with slip effects is solved using three approximate methods based on three different geometrical configurations. These methods provide an alternate approach to the conventional methods of solving two dimensional problem. With the help of these methods we are able to have an idea about the geometrical effects of curved channel on the flow which otherwise are not observed by using other methods. Expressions for velocity and pressure gradient are calculated for each method. Weight functions are calculated for all three methods and are evaluated for different values slip parameter $\gamma$. It is observed $F_{1}, F_{2}$ and $F_{3}$ functions are influenced by $\gamma$ and have smaller values than those in the case of no-slip.

To study the effect of $\gamma$ on flow pattern in arteries having stenosis an idealized stenosis geometry is considered and resistance to the flow and shear stress are calculated. It is noticed that as we increase the value of $\gamma$ resistance to the flow in the stenosed region decreases as expected. However with increasing the values $\gamma$ and $\lambda_{1}$ the shear stress increase in stenosed region. We can conclude that the over all effect of $\gamma$ is to decrease the resistance to the flow and thus to reduce the abnormalities due to irregular boundaries. It is also found that our results are more general as we can recover

- solution obtained by Langlois as $\gamma \rightarrow 0$ and by setting $\alpha_{1}=\alpha_{2}=0$.

- solution obtained by Siddiqui et al as $\gamma \rightarrow 0$.

We hope that this investigation may be helpful in evaluating the performance of various prosthetic devices that ultimately may be implemented into living system.

\section{Acknowledgements}

The authors are very much thankful to the anonymous reviewers for their valuable comment towards the improvement of this article. The corresponding author is very thankful to Higher Education Commission (HEC) of Pakistan for funding in her higher studies under the 5000 indigenous scholarship scheme Batch-IV. 


\section{REFERENCES}

[1] D. F. Young. Effect of a time dependent stenosis on flow through a tube, Journal of Engineering for IndustryTransactions of the ASME , Vol. 90, 248-254,1968.

[2] D. F. Young, F. Y. Tsai. Flow characteristics in model of arterial stenosissteady flow, Journal of Biomechanics, Vol. 6, 395-410, 1978.

[3] D.A. McDonald. On Steady Flow through Modeled Vascular Stenosis, Journal of Biomechanics, Vol. 12, 13-20, 1979 .

[4] V. Vand. Viscosity of Solutions and Suspensions, The Journal of Physical and Colloid Chemistry, Vol. 52, 277$321,1948$.

[5] A.L. Jones. On the Flow of Blood in a Tube, Biorheology, Vol. 3, 183-188, 1966.

[6] Y. Nubar. Effects of Slip on the Rheology of a Composite Fluid: Application to Blood Flow, Rheology, Vol. 4, 133-150, 1967.

[7] P. Brunn. The Velocity Slip of Polar Fluids, Rheologica Acta , Vol. 14, 1039-1054, 1975.

[8] G. Bugliarello, J.W. Hayden. High Speed Microcinematographic Studies of Blood Flow In Vitro, Science, Vol. 138, 981-983, 1962.
[9] L. Bennet. Red Cell Slip at a Wall in vitro, Science, Vol. 155, 1554-1556, 1967.

[10] N. Rashevsky. A Problem In The Mathematical Biophysics Of Blood Circulation: II. Relation Between Pressure And Flow Of A Viscous Fluid In An Elastic Distensible Tube, Bulletin Of Mathematical Biophysics, Vol. 7, 25-33, 1945 .

[11] G. W. Morgan. On The Steady Laminar Flow Of A Viscous Incompressible Fluid In An Elastic Tube, Bulletin Of Mathematical Biophysics, Vol. 14, 19-26, 1952.

[12] W. F. Langlois. Creeping viscous flow through a two dimensional channel of varying gap, Proc. Third U.S, Nat. Gong. Appl. Mech, 777, 1964.

[13] A. M. Siddiqui, T. Haroon, Z. Bano, S. Islam. Pressure Flow of a Second Grade Fluid Through a Channel Of Varying Width With Application to Stenosed Artery, International journal of Biomathematics. Vol. 06, 1350016, 2013 DOI: 10.1142/S1793524513500162.

[14] R. I. Tanner. Plane Creeping Flow of Incompressible Seond-Order Fluids, The physics of fluids. Vol.9, N. 6, 1246-1247, 1966. 